\title{
Research on Fast Access in Power System Simulation Base on Oracle
}

\author{
Zheng Huankun \\ North China Electric Power University \\ Baoding 071003, China
}

\author{
He Zhanbin \\ North China BAODING Electric Power VOC.\&TECH. \\ College \\ Baoding 071051,China
}

\begin{abstract}
In order to meet the request of fast access of power system simulation software base on Oracle, two methods based on OCI (Oracle Class Interface) technology are presented in this paper. The method of Binding array is used for reading data and the method of direct path load is used for writing Oracle database.Compared with prevalent ADO or ODBC technology, OCI has more efficient in writing or reading bulk data from Oracle. With these methods, it can meet the real time requirements of power system simulation. OCI has greater scope comparing with other methods, and OCI is more suitable for power system simulation requirements. At last, a simulation example and partial codes are provided.
\end{abstract}

Keywords-Power system simulation, Database, Real time, OCI, Batch deal

\section{INTRODUCTION}

With the development of electric power systems and electronic computer technology all kinds of power system monitoring and simulation program generally introduce the concept of database, and play a cole position in these systems.In the power system the database should be primarily used to store power system operation data, analyze data and various historical data, such as generator, transformer, track and other equipment, electrical parameters of operation parameters and the power of limits, relay protection and automatic device setting value etc[1].For simulation system will save the initial status, fault setting, calculation process and calculation results, etc.The simulation calculation of power system database management besides should pay special attention to quickness besides accuracy, flexibility and security. The requirement of database query and renewal speed is more and more faster.[2]

Oracle database with its outstanding performance in the power system applied widely, but Oracle database application development languages and development mode of performance for Oracle access interface has an important influence on it's effect[3]. Most of the simulation system using ODBC, DAO, OLE DB as middleware to access Oracle database, but with the fault of programming complex, low agility and low rate. Many methods to access database and whether we can make full use of Oracle database advanced features is shown as table 1-1.This paper puts forward a method that using OCI technology in Oracle database access. When reading and writing large quantities of initialized data or results OCI directly binding fast loading method is used. The practice has proved that using OCI technology can effectively improve the performance of database access, powerful functions, rapid mass data reading and writing performance and can well satisfy the power system simulation calculation .

TABLE I. COMPARISON OF ORACLE FUSION MIDDLEWARES

\begin{tabular}{|c|c|c|c|c|c|}
\hline Items & Performance & universal & $\begin{array}{c}\text { programmi } \\
\text { ng } \\
\text { complexity }\end{array}$ & $\begin{array}{l}\text { control } \\
\text { degree }\end{array}$ & $\begin{array}{c}\text { advance } \\
\text { d } \\
\text { features }\end{array}$ \\
\hline OCI & very high & Oracle & complex & high level & yes \\
\hline ODBC & low & relational & $\begin{array}{l}\text { relatively } \\
\text { simple }\end{array}$ & high level & no \\
\hline $\begin{array}{c}\text { Pro } \\
\mathrm{C} / \mathrm{C}++\end{array}$ & high & Oracle & simple & low level & yes \\
\hline OLE DB & above normal & all & complex & low level & no \\
\hline ADO & middle & Oracle & $\begin{array}{c}\text { relatively } \\
\text { simple }\end{array}$ & high level & no \\
\hline $\mathrm{OO} 4 \mathrm{O}$ & above normal & Oracle & middle & high level & yes \\
\hline JDBC & above normal & most & $\begin{array}{c}\text { relatively } \\
\text { simple }\end{array}$ & low level & no \\
\hline
\end{tabular}

\section{OCI READ DATABASE METHOD}

\section{A. Overview of OCI method}

OCI is a group of application programming interface (API) provided by ORACLE company in order to access ORACLE database. OCI provides bottom interface of database, reduces the layers of encapsulation. Therefore the mode of accessing Oracle database is flexible and the controlling is very strong. So it can offer direct links between programs and database and it's efficiency is very high. OCI has the following features:

Support ORACLE object-oriented technology fully;

Height control application development;

Support advanced language development OCI program;

Support Dynamic SQL;

Oracle development tools OCI popular support;

Through callback technology realize dynamic binding and definitions;

Enhance the application of manipulation language (DML) language;

Support most operating systems, and have wide application range;

With the development of electric power system, Parallel computing gradually replace stand-alone calculation, cloud computing as a new parallel computing method is expected 
to get application and development in power system. This condition requires database system not only have the rapid reading and writing data but also support multiple server system.

The references [4] put forward a method of using OCL writing ORACLE database, this method can greatly improve the speed of writing results to database in power system simulation. But the method only has local function, and can not apply to multiple service system, another encapsulation of OCI reduces the writing efficiency. In addition, now monitoring and simulation systems usually just pay attention to data about people read speed, speed for database rarely mention.

\section{B. The method of access database with $O C I$}

For a given power systems, the initial condition, equipment parameters and operation parameters is fixed.

Therefore, the power system simulation program has its own distinct features when reading and writing the database: the quantity of data need to read and write is huge, the types of data is complex; the demand speed of reading and writing is high; the format of Reading and writing data is fixation; the items and rows of database is fixed. In the power system simulation program initialization not only need to read each generator, transformer, lines and load parameters ,the section of simulation calculation data etc, but also need to read step length,fault informations from the database and so on. After a step, calculation results and running state changes and other information need to write back to database.

1) The Reading of database method with $O C I$

Fully considering the characteristics of power simulation system, reading data from database by binding array method. This method is applicable to the program initialization, simulation results playback, run after breakpoint.

For these variables who need to be assigned values, first establish the mapping relationship between their address and the result set of corresponding SQL statements. Put their storage address in bound to the SQL statements directly before the query database.

Recordset get by executing the SQL statements directly deposited in the corresponding variables through the mapping relationship to the data, avoiding to treat the SQL statement results one by one in order to assign values to variables. Through these method, query time will be shorter and initialization efficiency also become higher.

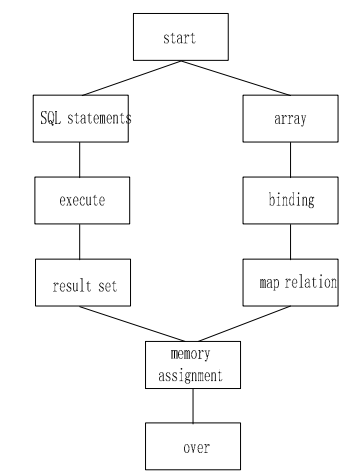

Figure 1. Reading data flow chart

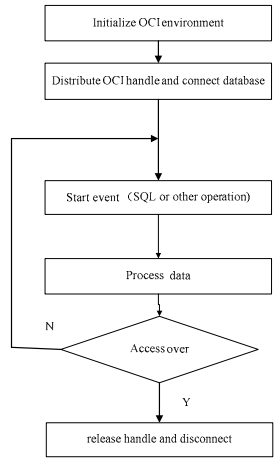

Figure 2. programming steps flow chart

\section{2) Direct path load method}

OCI methods have two loading data method: conventional load and direct path load[3]. Conventional loading executives SQL INSERT statements with bind variables and inserts data to the table of Oracle database. While direct path loading uses the characteristics of Oracle array interface to format the data into Oracle data block, then directly writes these block into database table. This method will not use caching mechanism, So it bypasses the RDBMS treatment, reduces the overhead of Oralce database.

In the process of simulation results of each step should be stored in a database simulation results in order to prepare analysis or playback. So the mass data need to write into Oracle database frequently. The speed of writing must be quicker, and at the same time, the format of data needed to be written is fixed. According to the above characteristics, the direct path loading method is more appropriate.

First, the speed of direct path loading is very high than conventional loading and other methods, because direct path loading direct will directly put the data into internal data stream format, Oracle database reducts these data back to item data and directly writes these data into table. conventional load uses buffer mechanism and unders the control of RDBMS, so it's speed is lower.Secondly, Using the direct path loading, the data loading SQL statement does not need to be performed beause these work has been done in the initialization. And the loading paths and mapping relation in simulation program has been completed.

From the above, the direct path method has obvious advantages when writing simulation results into Oracle database. The advantage is that this method will not compete database resources with other users, especially will not occupy CPU times; data loading time becomes shorter and the loading speed becomes higher.Figure 3 is a direct path loading data flow diagram.

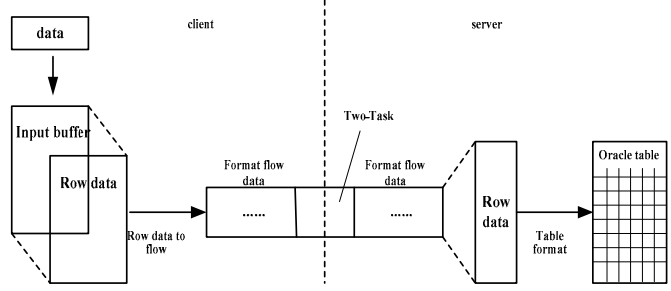

Figure 3. direct path loading data flow diagram 


\section{PRogramming ReAlize}

The conventional steps OCI programming is shown as figure 2. General power system simulation is as follows: power system simulation programs read the initial data, simulation settings of initial conditions. Using these data and the model of power system the program is running. Results of every step calculation will be deposited into database; When Simulation ends, we will study the whole process data.

\section{A. Reading database}

Using OCI binding array method to read database, for example, reading the SWITCH state. Refer figure 1, the first step we should initialize the environment, prepare SQL statements, bind array with SQL statements and so on. Main core statements are following:

OCIDefineByPos(stmtp, \&hDefine, errhp, 1, \&SW[0].no,sizeof(SW[0].no), SQLT_INT, NULL,NULL,NULL,OCI_DEFAULT); OCIDefineByPos(stmtp,

$\&$ hDefine,errhp,2,\&SW[0].state,sizeof(SW[0].state), SQLT_STR,\&sb2aInd[0],NULL,NULL,OCI_DEFAUL $\mathrm{T})$;

OCIDefineArrayOfStruct(hDefine, errhp, 16,0,0,0);//bind array OCIStmtExecute(svchp,stmtp,errhp,0,0,NULL,NULL,OCI_DEFAULT);//e xecute SQL

OCIStmtFetch(stmtp,errhp,1356,OCI_FETCH_NEXT,OCI_DEFAULT);// get results

\section{B. writing database}

Each step simulation results would include at least generators, line, bus and node, etc, such as the active and reactive power, rotate speed, power-angle of generator and so on; the active and reactive power of first and end line etc. These data are stored in OUTPUTNODE, UTPUTLINE, OUTPUTLOAD, OUTPUTGEN four tables. Current step results must be stored into database completely before the next step results come, otherwise, the following data will replace the current results. If this happens, the simulation results will not be correct. The data quantity of each step results is large and needed to be written into database less than one step. The writing speed must be very high.OCI direct route loading methods in this respect has obvious speed advantage. Main core statements are following:

//First put data into data flow. Specific code omit.

OCIDirPathColArrayToStream(dpca,dpctx,dpstr,errhp,ro wnum,rowoff);

//add array to data flow

OCIDirPathLoadStream(dpctx,dpstr,errhp);// load data

OCIDirPathFinish(dpctx,errhp);// over direct path load

\section{EXAMPLE ANALYSIS}

This paper example test environment for:

Hardware environment: the CPU Intel (R) Core: (TM) 2 $2.0 \mathrm{GHz}$

Memory: $2 \mathrm{G}$.

Hard disks: 250G 7200 RPM;

Software environment: operating system: Windows 2003 Server;

Database: Oracle8i.

Programming environment: $\mathrm{vc}++6.0$.
This example simulation is a province power system, simulation results include: 120 generators data, 806 routes data, 636 loads data and 5135 nodes data, aggregate data size for 97348 bytes;

Time1 is defined as the time accessing all data from SWITCH table (total 6876 recorded); Time 2 and Time 3 is the same define with Timel while the size of table added by 1000 rows ordinally; Among them, Time1,Time2 and Time 3 are the average of 500 times tests. Time in Tab. 2 is defined as the time to read data from the database and assign them to variables. Time in tab. 3 is defined as the time for writing the same array data into database.

TABLE II. COMPARISON OF READING SPEED

\begin{tabular}{|c|c|c|c|}
\hline items & Time1 & Time2 & Time3 \\
\hline ADO & 53.84 & 62.69 & 69.87 \\
\hline OCL & 91.18 & 103.58 & 118.34 \\
\hline OCI & 10.68 & 12.77 & 13.30 \\
\hline
\end{tabular}

TABLE III. COMPARISON OF WRITING SPEED

\begin{tabular}{|c|c|c|c|}
\hline items & Time1 & Time2 & Time3 \\
\hline ADO & 9390 & 1049 & 11421 \\
\hline OCL & 39.11 & 45.90 & 47.95 \\
\hline OCI & 28.14 & 30.99 & 35.75 \\
\hline
\end{tabular}

From the Tab.2 can be seen that the speed of binding array method is almost 5 times of the speed of ADO method and almost 9 times of the speed of OCL method when reading the same rows for the same database table. From the Tab. 3 can be seen that the speed of OCI direct path loading method is almost 300 times of the speed of ADO method and almost 1.4 times of the speed of OCL method when writing the same rows for the same database table.

OCI technology has faster reading and writing speed, it can also use the advanced functions, the applied range is very wide and programming is more agile. This method is more suitable for frequent access to Oracle database, and mass data access database form relatively fixed power system simulation system.

\section{SUMMARIES}

This paper puts forward OCI method to solve the problem of access Oralce database in power system simulation software. The mehod of binding array is used to enhance the reading speed and the direct path loading method is used to solve the problem of writing Oralce database speed bottlenecks. Through the analysis and comparison of all kinds of methods we can see that the two method have obvious advantages than other methods. Furthermore, these methods can also be used in other monitoring, simulation systems which have higher demand for real-time.

\section{REFERENCES}

[1] Gu Yi; Zhao Yuzhu; Zhang Guowei. A SURVEY OF THE DISPATCHER TRAINING SIMULATOR AND ITS APPLICATION IN CHINA [J]. Automation of Electric Power Systems, 2002, 26(13): 60-62, 65. 
[2] DONG Zhao-xia; YANG Feng; GAO Jian-jun; FAN Dou; HE Nanqiang. Design and Implementation of the Database in Network DTS [J]. Hubei Electric Power, 2003, 27(3) : 6-9.

[3] DONG Zhao-xia, YANG Feng,GAO Jian-jun . Design and Implementation of the Database in Network DTS[J]. Hubei Electric Power, 2003, 27(3): 6-9.

[4] WANG Jun-zhuang; CHANG Xian-rong;. Research on quick access in Oracle database based on OCL technology [J] Power System Protection and Control,2009,9

[5] Liu yong-ning. Direct Path Loading in Oracle $8 \mathrm{i}$ with OCI[J]. Computer Applications 2002, 22(4) : 88-89.

[6] TAN Hai-long; CHANG Xian-rong. Study on active memory database in DTS $[\mathrm{J}]$. Journal of North China Electric Power University, 2005, 32(2) : 71-74.
[7] NIE Hong-mei; ZHAO Jian-min. Research of Optimum Query Technology on Clob Big Segment in Oracle Database [J]. Computer Technology and Development, 2006, 16(8) : 97-102.

[8] Core Lab. Oracle Class Library for C++ 3.00.0.1[DB] 。 http://crlab.com/ocl/,2005.

[9] FU Qiang; ZHANG Xi-shu; Design of Power Quality Monitoring System Based on Virtual Instruments and Database Technology[J]. Electrical Measurement \& Instrumentation,2010-03

[10] JIANG Yue ; A Research on Optimization of Database Query Method[J]. Computer Knowledge and Technology, 2011-05

[11] Luo jian. Research of Real-Time Transaction Processing on DTS[J]. Huazhong University of Science \& Technology, 2006

[12] ZHOU Li Ping ;ZH U Li Min; The Factors of Distributed Data Base Systems Data Accessing Efficiency[J]. MICROCOMPUTER DEVELOPMENT, 1998-2 